\title{
EDUCATION AND TRAINING Incivility and the clinical learner
}

\author{
Authors: Laura JE Cheetham ${ }^{A}$ and Christopher Turner ${ }^{B}$
}

The impact of incivility in terms of individual and team performance in clinical environments is increasingly acknowledged and supported by a growing evidence base. However, clinical environments are not just areas where patient care is delivered, they are also rich, key learning arenas for healthcare professionals. To date, the potential impact of incivility in clinical environments on healthcare professional learning and development has not been comprehensively explored. This article provides an overview of the physiological mechanisms that inhibit learning and memory recall in individuals experiencing or observing incivility and social stress. It establishes a clear need for focus on the impact of incivility on clinical learners and educators and further evidence for the need for clinical environments in which civility is firmly rooted into the pervading culture.

KEYWORDS: Incivility, learning, prefrontal cortex

DOI: $10.7861 /$ fhj.2020-0008

\section{Introduction}

There is increasing acceptance of the impact of incivility in clinical workplaces in terms of reduced performance of both the recipient and bystanders. ${ }^{1,2}$ What has been less well explored is the impact that incivility has on one key area in healthcare learning. It is a requirement that all doctors engage in lifelong learning with a significant portion of this knowledge development occurring in the clinical workplace, where it is generally done in a Socratic or didactic fashion. From the perspectives of professional performance and patient safety, it is in the interest of teachers, learners and patients that we make this education as efficient and effective as possible.

\section{Could civil environments promote learning and recall}

Over the last 20 years, the evidence base around the impact of incivility on individual and team performance has grown to include healthcare environments, with simulation-based studies showing a statistically significant decrease in performance even when the

Authors: ${ }^{A}$ general practitioner registrar, Worcester Acute Hospitals NHS Trust, Worcester, UK; ${ }^{\mathrm{B}}$ consultant in emergency medicine, University Hospitals Coventry and Warwickshire NHS Trust, Coventry, UK and co-founder of Civility Saves Lives, UK. rudeness is mild. Incivility manifests itself as stress (arousal), steals from our working memory and extends beyond the direct recipient to bystanders and patients. The behaviours can be verbal and nonverbal; they extend from simple rudeness and undermining, and can continue all the way through to overt bullying.

There is now a growing body of evidence that stressful learning environments, including where the stress is received as being generated by the educator, can both directly and indirectly impact on the ability of learners to develop effectively.

Therefore, could incivility, as a specific form of perceived stress, be a detriment to learning?

Medical education relies on the delivery of effective, high-quality learning within clinical environments, and is a core component of quality assurance for all healthcare professionals. What elements make up a high-quality learning environment where the potential for learning is maximised? Studies suggest that there is a need for a psychologically safe learning environment without exposure to unnecessary stressors, to strengthen human memory. ${ }^{3,4}$

Learning underpins all aspects of medical professionalism in order for individuals and clinical teams to consistently and continually deliver the safest and highest-quality patient care. ${ }^{5}$ It appears, increasingly, that hand-in-hand with effective learning is a need to maintain learner wellbeing. Psychologically safe environments are open, allowing for creative conflict where individuals feel able to express opinions and where there is less time spent on 'image management'. When staff feel psychologically safe they are able to ask the questions that are troubling them and to raise issues that concern them. This has obvious implications for both the learning of the individual and for patient safety. Civility fosters a psychologically safe environment by reducing the negative responses to questions posed by learners and, when they are in turn questioned, to incorrect responses from the learners.

\section{Mechanisms through which perceived hostile environments impact learning}

The mechanisms by which incivility can damage wellbeing are various in learners: inciting stress, unhappiness, fear, anxiety, a heightened threat response and sleep disturbance. ${ }^{6-10}$ Incivility results in disruption of the educator-learner relationship by creating unwanted distractions in busy clinical learning environments and so loss of concentration on the task at hand and, for others, loss of self-esteem. All of these factors are known to impact on the quality of learning. 3,12-17.

There is a body of evidence suggesting that stress impairs learning (ie the process of receiving and encoding information and recalling memories to build on) other than that pertaining directly to coping or escaping from the stressor. ${ }^{18}$ Research also 
suggests that while emotionally driven information resulting from a traumatic event may be retained far more effectively than 'neutral' information, building further knowledge based on prior information recall is substantially impaired. ${ }^{4,19}$ This means that stress may enhance memories of emotional material related to the stressful context, but any other information related to that event is typically not well remembered, as stress may impair the encoding of neutral information. Clinical knowledge is less likely to be emotion-based than neutral, which means that factual knowledge retention (declarative memory) may be poorly promoted in a stressful learning environment such as one caused by incivility.

There is evidence to show that raised adrenaline levels (and possibly cortisol levels) and activation of an acute stress response, including increased activity in the amygdala, reduce embedding in short- and long-term memory. ${ }^{20}$ In contrast, there is some evidence that raised stress (arousal) levels in the 20 minutes prior to a learning event may increase recall. This latter finding is based on mild to moderate stress levels in schools and may not be generalisable to adult workplace-based learning, such as perceived hostile questioning in medicine. Medical students report greater than average stress levels, with interactions with consultants being rated as the most stressful experience during their placements. ${ }^{21}$ Stress signalling pathways impair prefrontal cortex function reducing the ability to learn new concepts and build new learning around previously encountered and embedded concepts. ${ }^{3,22,23}$ In addition, induced stress moves brain activity to affective brain areas, executive areas and the hippocampus; this could impair attention, assimilation and accurate memory encoding. ${ }^{24}$ Stress has been shown to have a substantial effect on memory in a time dependent way; longer or more frequent exposure to stress has more of a detrimental effect on learning and memory. ${ }^{25}$ Recurrent exposure to perceived stressful situations has been shown to result in changes to dendritic length, branching and spine loss in the prefrontal cortex. This is important for working memory and the longer-term memory encoding process and leads to expansion of dendrites in the amygdala instead of longer-term memory pathways. ${ }^{22}$

There are other potential effects of stress on the recall of established knowledge learnt in the past if this information is recalled under stressful conditions. The process of retrieving pre-existing information under stress is thought to make this knowledge more unstable and fluid prior to reconsolidation. ${ }^{26}$ Thus, previously learnt knowledge can be impaired or distorted if done so under stressful conditions, losing or reducing the amount or quality of prelearnt material. Given that doctors are required to be lifelong learners, consistently building on knowledge already acquired, the impact of stress caused by incivility on pre-existing knowledge is worthy of note and of further investigation. The mechanism of this is thought to be most likely due to the effects of stress on the hippocampus and prefrontal cortex. ${ }^{27,28}$ Therefore, the more frequently clinicians are immersed in an uncivil environment, the more their learning and development slows.

Particular forms of learning that may be even more negatively affected by incivility, in terms of the degree of information and knowledge learnt in a stressful environment, are tacit learning and multisequencing learning. ${ }^{3}$ Tacit learning is a cornerstone of identity as a doctor, of our practice and of our professionalism, it is the mechanism by which we absorb and integrate medical culture, communication skills and a plethora of other factors which constitute the 'hidden curriculum'. Multisequencing learning allows medical practitioners to learn new practical and procedural skills among other elements of their development. There is evidence also that information recalled from memory during stressful situations might be 're-learnt' or modified incorrectly directly as a result of stress exposure. ${ }^{3}$ It is possible that the stress caused by incivility in our clinical learning environments not only impair our efforts to learn but also erode knowledge we have already acquired.

Studies also suggest directly that incivility within the clinical educator and individual learner relationship results in impaired learning. Suggestive causative reasons are the development of mistrust; a negative and ineffective relationship; a sense of feeling 'put down'; loss of respect; disempowerment; stress and fear development; and erosion of confidence and effective communication - in summary, the creation of a negative and dysfunctional learning environment. ${ }^{17,29,30}$

Research regarding nursing learners is more extensive than for physician learners, with one study going so far as to state that 'Incivility is the largest documented barrier to student-faculty relationships in nursing and is an element associated with students not achieving desired academic outcomes. ${ }^{31}$

There are further potential ramifications of incivility for clinical education including implications for the next generation of learners. Potentially, through role modelling, the approach to delivering teaching in an uncivil way could be perpetuated if learners assume that this approach is the inherent, expected and accepted way that clinical education should be delivered in their communities.

\section{Avenues for further research}

There is currently a paucity of research directly quantifying the impact of incivility by educators or peers on learning when in a medical environment. Additionally, there is little understanding of whether learning is affected in different ways depending on the format of the clinical educational session; for example, bedside teaching versus practical skill training versus debrief teaching.

Investigating the underlying neural mechanisms that may be involved in the impact of incivility during a teaching encounter on memory and learning would lead to greater understanding of how educators can adapt their approaches to maximise the value of clinical learning.

Further research could, in the simulated environment, directly investigate the retention of learning in controlled simulated clinical learning environments versus clinical teaching scenarios with different forms of stress present, including incivility. This has the potential to help separate the impact of different stressors and aid clarity regarding which of the potential stressors that can occur within a complex clinical environment have a negative impact on learning, and to what extent they do this. This would provide valuable insights both for the clinical teacher and the learner.

\section{Conclusion}

There is a growing body of evidence indicating that incivility has a negative impact on learning and recall, though the evidence is stronger for recall than learning and the impact of stress on learning may vary substantially depending on the emotional and stress-related nature of the information itself. This evidence sits alongside our knowledge that incivility reduces performance in complex clinical environments. On balance, we believe that the 
benefits of civil behaviours outweigh any benefits of increased stress for learners. It is key that the link and potential impact of behaviours in clinical learning environments are explored further. If incivility in the learning relationship has negative implications for learning, then setting the compass for educator-learner interactions firmly towards civility would be a simple and effective way to enhance and accelerate the development of doctors, and other clinical learners, and to help those in medicine achieve the potential that they are capable of.

\section{Conflicts of interest}

Laura Cheetham and Chris Turner are members of Civility Saves Lives campaign group. However, this article was entirely independent work by the authors and did not receive any input or review prior to publication from Civility Saves Lives members.

\section{References}

1 Katz D, Blasius K, Isaak R et al. Exposure to incivility hinders clinical performance in a simulated operative crisis. BMJ Qual Saf 2019;28:750-7.

2 Riskin A, Erez A, Foulk TA et al. The impact of rudeness on medical team performance: a randomized trial. Pediatrics 2015;136:48795.

3 Edmondson A, Lei Z. Psychological safety: The history, renaissance, and future of an interpersonal construct. Annu Rev Organ Psychol Organ Behav 2014:1:23-43.

4 Klatt EC. The human interface of biomedical informatics. J Pathol Inform 2018:9:30.

5 General Medical Council. Continuing professional development: guidance for all doctors. GMC, 2002.

6 Beattie L, Griffin B. Day-level fluctuations in stress and engagement in response to workplace incivility: A diary study. Work \& Stress 2014;28:124-142.

7 Clark C. Student perspectives on faculty incivility in nursing education: an application of the concept of rankism. Nurs Outlook 2008:56:4-8.

8 Luparell S. Faculty encounters with uncivil nursing students: An overview. J Prof Nurs 2004;20:59-67.

9 Luparell S. The effects of student incivility on nursing faculty. J Nurs Educ 2007:46:15-9.

10 Altmiller $\mathrm{G}$. Student perceptions of incivility in nursing education: Implications for educators. Nurs Educ Perspect 2012;33:15-20.

11 Sprunk EA, LaSala KB, Wilson VL. Student incivility: Nursing faculty lived experience ] Nurs Educ Pract 2014:4:1-12.

12 Caza BB, Cortina LM. From insult to injury: Explaining the impact of incivility. Basic and Applied Social Psychology 2007;29:335-50.

13 Craik F, Govoni R, Naveh-Benjamin M et al. The effects of divided attention on encoding and retrieval processes. J Exp Psychol Gen 1996:125:159-80.
14 Fernandes MA, Moscovitch M. Divided attention and memory: Evidence of substantial interference effects at retrieval and encoding. J Exp Psychol Gen 2000;129:155-76.

15 Iidaka T, Anderson N, Kapur $S$ et al. The effect of divided attention on encoding and retrieval in episodic memory revealed by positron emission tomography. J Cogn Neurosci 2000;12:267-80.

16 Sauer PA, Hannon AE, Beyer KB. Peer incivility among prelicensure nursing students: a call to action for nursing faculty. Nurse Educ 2017:42:281-5

17 Rawlins L. Faculty and student incivility in undergraduate nursing education: an integrative review. J Nurs Educ 2017;56:709-16.

18 Schwabe L, Wolf OT. Learning under stress impairs memory formation. Neurobiol Learn Mem 2010;93:183-8.

19 Schwabe L, Vogel S. Learning and memory under stress: implications for the classroom. Npj Science of Learning 2016;1:16011. www.nature.com/articles/npjscilearn201611.pdf

20 Sandi C. Memory impairments associated with stress and aging. In: Bermúdez-Rattoni F (ed), Neural plasticity and memory: from genes to brain imaging. Boca Raton: CRC Press/Taylor \& Francis, 2007. www.ncbi.nlm.nih.gov/books/NBK3907

21 Firth J. Levels and sources of stress in medical students. Br Med J (Clin Res Ed) 1986;292:1177. www.bmj.com/content/292/6529/1177

22 Arnsten A. Stress signalling pathways that impair prefrontal cortex structure and function. Nat Rev Neurosci 2009;10:410-22.

23 Joels M, Pu Z, Wiegert $O$ et al. Learning under stress: How does it work? Trends in Cognitive Sciences 2006;10:152-8.

24 Khalili-Mahani N, Dedovic K, Engert V et al. Hippocampal activation during a cognitive task is associated with subsequent neuroendocrine and cognitive responses to psychological stress. Hippocampus 2010;20:323-34.

25 Quaedflieg CW, Schwabe L, Meyer T, Smeets T. Time dependent effects of stress prior to encoding on event-related potentials and $24 \mathrm{~h}$ delayed retrieval. Psychoneuroendocrinology 2013;38:3057-69.

26 Nader K, Hardt O. A single standard for memory: the case for reconsolidation. Nat Rev Neurosci 2009;10;224-34.

27 Schwabe L, Nader K, Pruessner JC. Reconsolidation of human memory: brain mechanisms and clinical relevance. Biol Psychiatry 2014;76:274-80.

28 Sandrini M, Censor N, Mishoe J, Cohen L. Causal role of prefrontal cortex in strengthening of episodic memories through reconsolidation. Curr Biol 2013;23:2181-4.

29 Wagner B, Holland C, Mainous R et al. Differences in perceptions of incivility among disciplines in higher education. Nurse Educ 2018;44:265-9.

30 Mott J. Undergraduate nursing student experiences with faculty bullies. Nurse Educ 2014;39:143-8.

31 Ingraham KC, Davidson S], Yonge O. Student-faculty relationships and its impact on academic outcomes. Nurse Educ Today 2018;71:17-21.

Address for correspondence: Dr Christopher Turner, University Hospital, Clifford Bridge Road, Coventry CV2 2DX, UK.

Email: chris.turner@uhcw.nhs.uk 